\section{A microscope you can use with all your PPE}

According to Dr Jenner Argueta DDS, MSc, a senior lecturer in endodontics, the New Seiler Promise Vision 3D is ergonomically the best microscope currently on the market and can also help protect you from aerosols and infectious respiratory droplets making contact with the conjunctiva.

Seiler's New Promise Vision 3D Surgical Microscope allows the dental practitioner to have an ergonomically organised workflow, to maintain the safest possible working distance, and to obtain superb illumination, magnification and crystal-clear images without losing the perception of the clinical environment.

All of this can be achieved even when wearing all the personal protective equipment needed at the moment. In simple words, it is the perfect ally for being able to perform high quality dental treatments and working protected from any source of contamination.

For further details and opportunity to see this in practice contact Seiler Instruments' Representative for EMEA, Alec Hilton, on ahilton@ seilerinst.com, visit www.seilerinst. com and see https://www.youtube.com/ watch?v=zCvlyAUrczs.

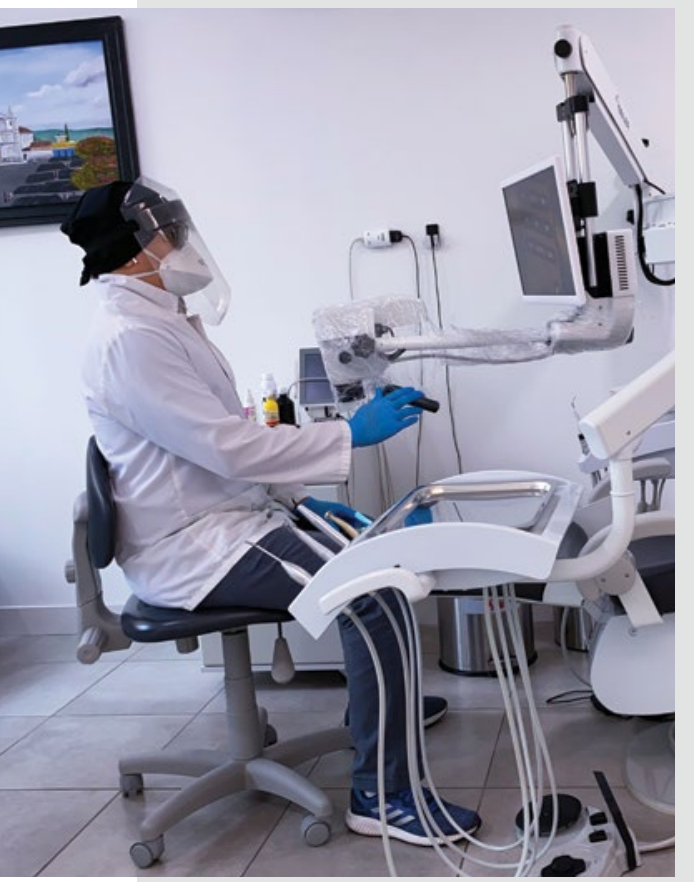

\title{
A complete set for professional tooth cleaning
}

Young Innovations and NSK present SMILEBOX, a complete set for professional tooth cleaning. The products perfectly match and complement each other. Each box contains a prophy contraangle handpiece, prophy cups, prophy paste, disclosing solution and parotid patches.

2Tone Solution reveals plaque with an advanced multicolour formula. It turns new plaque red and older blue, showing patients the areas they consistently miss while brushing the teeth. This not only serves to monitor patients; it also enables individual recommendations for dental hygiene at home.

The DryTips parotid patches are placed on the inside of the cheek directly in front of the largest salivary gland. The cellulose pads keep the work area dry during treatment, are far more absorbent than cotton rolls, but take up less space. They therefore particularly facilitate procedures in the back of the oral cavity.

The Young Elite Cup is a perfect match for the NSK stainless steel contra angle handpiece FX57m. The short polishing cup in combination with the ultra-small head enables easy access to all quadrants. The screw-type cup adapts neatly to the contra angle, while the soft rubber adapts completely to the tooth surface. While the internal ridges remove plaque, the external ridges support interproximal cleaning and reduce splatter.

The Young mint polishing paste with baking soda ensures the brilliant shine. It is free of fluoride and gluten and packed in hygienic single-unit doses. Its adhesive consistency reduces splatter, the non-abrasive baking soda removes discoloration and in one single step also achieves amazing lustre. For more information about SMILEBOX visit www.youngdental.eu/en/products_gb/ nsk-young-smilebox-en.

\section{Keeping people smiling}

TANDEX has added international standard ISO and PHD (passage hole diameter) numbers to the packaging of its FLEXI interdental brushes.

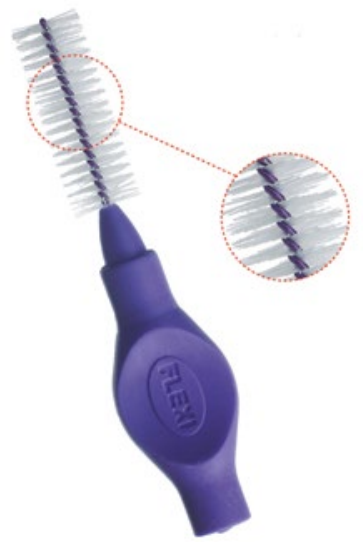

The ISO defines what PHD interval the brush can be squeezed into without deformation. PHD is based on the number of filaments per $\mathrm{cm}$, nylon thickness per $\mathrm{mm}$ and how hard the brush is twined. Dental practitioners can help patients find the right-size brush for them, meaning elevated daily cleaning.

This means improved oral health, general health and wellbeing too!

TANDEX wants to keep people smiling - find out more about all its products, for daily at-home preventive care, today.

For more information on Tandex's range of products, visit www.tandex.dk. 